\title{
Anabases
}

ANABASES Traditions et réceptions de l'Antiquité

$1 \mid 2005$

Varia

\section{François HARTOG, Régimes d'historicité. Présentisme et expériences du temps}

Pascal Payen

\section{(2) OpenEdition}

Journals

Édition électronique

URL : http://journals.openedition.org/anabases/1504

DOI : 10.4000/anabases. 1504

ISSN : 2256-9421

Éditeur

E.R.A.S.M.E.

Édition imprimée

Date de publication : 1 mars 2005

Pagination : 295-298

ISSN : 1774-4296

\section{Référence électronique}

Pascal Payen, « François hartog, Régimes d'historicité. Présentisme et expériences du temps », Anabases

[En ligne], 1 | 2005, mis en ligne le 01 octobre 2011, consulté le 22 septembre 2020. URL : http://

journals.openedition.org/anabases/1504; DOI : https://doi.org/10.4000/anabases.1504

Ce document a été généré automatiquement le 22 septembre 2020.

(c) Anabases 


\title{
François HARTOG, Régimes d'historicité. Présentisme et expériences du temps
}

\author{
Pascal Payen
}

\section{RÉFÉRENCE}

François HARTOG, Régimes d'historicité. Présentisme et expériences du temps, Paris, Seuil, coll.

«La Librairie du XXI ${ }^{\mathrm{e}}$ siècle ", 2003, 262 p.

$21 € /$ ISBN 2.02.059328.9

1 Les termes retenus par Fr. Hartog pour le titre de son dernier livre - Régimes d'historicité. Présentisme et expériences du temps - sonnent d'abord, dans leur singularité et par leur association déroutante, comme une invitation, lancée à lui-même et à ses lecteurs, à préciser ce que l'ouvrage n'est pas : ni une histoire des différents systèmes de périodisation du temps, qu'ils soient d'inspiration religieuse ou laïque, du livre de Daniel à Spengler, de Bossuet à Marx (p. 22-24); ni une analyse des conceptions du temps, en rapport avec l'écriture de l'histoire, pour prolonger l'enquête entreprise par Paul Ricœur, depuis Temps et récit (1983-1985) jusqu'à La mémoire, l'histoire, l'oubli (2000). Un régime d'historicité ne se présente pas non plus comme un système philosophique ou une "entité métaphysique " (p.118) à vocation englobante. Une fois précisé les tenants et les ressources, heuristiques et méthodologiques, de cette notion, le livre n'offrira pas plus une histoire continue et complète de chacun des régimes ni une liste de tous (p. 28).

2 Le point de départ de la réflexion est « la catégorie du présent dans ses rapports avec le passé et le futur» (p. 208-209). Le régime d'historicité peut être défini, dans un premier moment de l'analyse, comme la reconnaissance de la présence simultanée d'une dimension du passé, du présent et du futur, et des différentes manières de concevoir le rapport à ces trois temporalités. Mais la nécessaire délimitation des trois catégories ouvre sur un second moment, objet principal du livre, qui est l'exploration des modalités selon lesquelles sont articulés et pensés, les uns par rapport aux autres, 
passé, présent et futur. La notion de régime d'historicité peut ainsi aider à comprendre comment une culture, dans un espace et en un moment donnés, comme ceux des Maori des îles Fidji étudiés par Marshall Sahlins, par exemple, objet du premier chapitre, a organisé son rapport au temps, mais elle peut aussi s'appliquer à l'œuvre d'un individu, Chateaubriand ou Tocqueville. De cette notion, Fr. Hartog retrace la construction dans son propre cheminement intellectuel, depuis la première formulation explicite, dans un article de 1983 déjà consacré à Sahlins, en fait dès Le Miroir d'Hérodote (1980), d'où l'exilé-voyageur d'Halicarnasse ressort encore plus "historien" d'avoir été analysé comme ethnographe des autres, les Barbares en général, les Scythes en particulier. Sans que la démarche en soit alourdie, le lecteur se retrouve sans cesse au contact et à la croisée de l'anthropologie (Sahlins, Lévi-Strauss), de la philosophie préoccupée d'histoire (Foucault, Ricœur), de l'historiographie (De Certeau, Koselleck). L'apport le plus neuf du livre provient du choix d'explorer la validité de la notion, non en produisant un tableau général et historique des différentes régimes d'historicité, mais en faisant porter l'analyse principalement sur les «moments de crise du temps, [...] quand viennent justement à perdre de leur évidence les articulations du passé, du présent et du futur » (p. 27). Se trouvent mis en valeur les instants de rupture dans l'ordre du temps, "les limites et les seuils, les moments d'inflexion ou de retournement, les discordances » (p. 29). Un tel choix prend appui sur le concept de "brèche (gap) entre le passé et le futur ", introduit par Hannah Arendt dans La Crise de la culture (Between Past and Future) et défini comme « un intervalle dans le temps qui est entièrement déterminé par des choses qui ne sont plus et par des choses qui ne sont pas encore ", ainsi dans le Berlin d'après 1989 et la chute du Mur, ou au cours des années qui suivent la Révolution française. Ce parti pris est indissociable de la volonté de comprendre - selon le mot de Reinhart Koselleck - « comment dans chaque présent, les dimensions temporelles du passé et du futur [sont] mises en relation». Il explique la succession des chapitres et le rapprochement de quelques grandes figures que tout paraît éloigner, à commencer par le temps.

Le livre s'ordonne en cinq chapitres. Les trois premiers, sous le titre Ordre du temps 1 , offrent des exercices de " regard éloigné », en explorant ce que peuvent être, en dehors de la tradition occidentale (à Hawaï et à Fidji), « des formes de temporalité et des régimes d'historicité différents" (p. 47), puis avec les figures d'Ulysse et de Chateaubriand - déjà présentes, à des degrés différents, dans Mémoire d'Ulysse. Récits sur la frontières en Grèce ancienne (1996) -, ce qui conduit à l'émergence des régimes "ancien» et «moderne" d'historicité. Dans une analyse qui a beaucoup retenu l'attention, Fr. Hartog interprète les larmes que verse l'hôte des Phéaciens, un Ulysse alors dépourvu de son identité, lorsque l'aède Démodokos rappelle les exploits du héros, au chant VIII de l'odyssée, comme le signe d'une découverte et d'une prise de conscience de la notion de passé. Ulysse pleure, parce qu'il n'a pas les moyens de faire du présent d'autrefois, qu'évoque l'aède, un passé par rapport au présent de l'île de Schérie, et parce qu'il fait l'expérience douloureuse d'un abîme en lui-même, l'expérience du temps, d'une "distance de soi à soi", qui est "rencontre avec l'historicité » (p. 64). C'est le récit des événements de Troie, qu'il entreprend aussitôt devant ses hôtes phéaciens, qui lui permet de jeter un pont entre lui-même (au passé) et lui-même (au présent): le récit le conduit à la prise de conscience du temps historique. Ulysse redevient Ulysse en créant l'histoire d'Ulysse ; en ce sens, il est peutêtre la première esquisse de la figure de l'historien, comme voyageur et comme conteur. Du moins découvre-t-il la « passéité » du passé, le passé comme catégorie. 
Chateaubriand est ensuite privilégié dans l'analyse, parce qu'il est à la croisée des deux régimes d'historicité : l'« ancien » et le "moderne ». Selon le premier, condensé dans la formule cicéronienne de l'historia magistra vitae (De Oratore II, 36), l'histoire est pourvoyeuse d'exemples et de leçons; les modèles viennent du passé, qui permet de comprendre le présent et de prévoir l'avenir. Cette conception, dominante au moins depuis Varron, grand rassembleur et interprète des signes du passé de Rome, au dire de Cicéron encore (Académiques I, 3, 9), jusqu'à la veille de la Révolution, est adoptée par Châteaubriand dans la première édition de l'Essai historique, politique et moral sur les révolutions anciennes et modernes considérées dans leur rapport avec la Révolution française (1797). Puis, sous l'effet de 1789, s'instaure une conception «moderne» de l'histoire comme processus et progrès, issue des Lumières et tournée vers l'avenir. Désormais le passé est dépassé, "par position et par principe ", et c'est le futur qui éclaire l'histoire passée et donne son sens à l'avenir. Chateaubriand contribue, dans la seconde édition de l'Essai, en 1826, et dans les Mémoires d'outre-tombe, à instaurer ce changement. Le futur qui doit advenir, au nom de la Nation, du Peuple ou du Prolétariat, selon une liste qui n'est pas close, est aussi le point de vue selon lequel est reconsidérée l'histoire passée. Entre les deux régimes, Chateaubriand ne choisit pas; il reste dans la "brèche ", ne voulant renoncer à aucun, et c'est pourquoi il est certainement le personnage principal et exemplaire de ce livre. En cela, il se distingue des historiens «scientifiques » du XIX ${ }^{e}$ siècle pour qui le passé, objet d'étude, est séparé du présent; lui continue de voir le passé dans le présent. De là le constat, paradoxal et éclairant, fait par Fr. Hartog, que Chateaubriand peut " apparaître comme le frère lointain d'Ulysse " (p. 100) : ce dernier pleure de ne pouvoir formuler son historicité qu'il découvre; l'auteur des Mémoires en a fait le principe de son écriture, en explorant l'écart entre l'ancien et le nouveau régime d'historicité, décrits par lui comme les deux rives d'un seul et même fleuve, qu'il traverse tel un passeur, une figure de l'entre-deux, de la frontière.

5 Avec les chapitres 4 et 5, de la seconde partie, -Ordre du temps 2-, la perspective change. Non plus le "regard éloigné », mais la «contemporanéité", à partir de et autour de 1989. La chute du Mur - avec l'effondrement du régime communiste, qui met à mal la grande idéologie promouvant le futur au rang de point d'observation de l'histoire - est prise comme l'événement ou le symptôme marquant un changement de régime d'historicité. Désormais le présent fait loi. Deux mots, deux guides permettent d'explorer cette autre voie : la mémoire, à partir d'une étude de l'immense entreprise des Lieux de mémoire (1984-1993), dirigée par Pierre Nora et conçue comme une histoire du présent; le patrimoine, dont l'auteur offre une vue cavalière incisive, depuis la Rome de la fin de la République, jusqu'au dernier demi-siècle. L'un et l'autre participent d'une même crise du régime "moderne ». L'événement contemporain devient aussitôt son propre passé, en étant l'objet d'une commémoration immédiate, en tant qu'événement historique, ainsi de la chute du Mur ou du 11 septembre 2001. De même, le Patrimoine, qui a eu son " année ", en 1980, qui possède ses musées, sa "Direction " au Ministère de la culture, sa «loi » depuis 1993, ses instances internationales sous l'égide de l'UNESCO, témoigne de la faillite des deux autres régimes d'historicité : on préfère commémorer le passé, plutôt que l'étudier ; quant au futur, il est érigé au rang de patrimoine à sauvegarder, car pèse sur nous la menace que l'avenir ne soit pire que le présent. Au nom de la mémoire ou du patrimoine, on entre ainsi « dans un temps de prévalence du point de vue du présent», nommé ici "présentisme». Cette «historicisation immédiate du présent » est la marque de notre temps. Comme si le 
présent, omniprésent, devenait perpétuel, regardant à la fois vers le passé et vers le futur. Comme si l'histoire, dans ce régime d'historicité que nous forgeons hic et nunc, devenait mémoire.

6 Il est difficile de rendre compte d'un livre qui refuse les larges développements et les grandes machineries, par choix de méthode et par principe d'écriture. À quoi tient cependant son indéniable unité ? Ressaisir, dans la longue durée, les moments de brisure, les retournements, les zones de frontière, au sujet du rapport problématique entre les catégories de passé, de présent et de futur, permet de redonner vie et sens au temps, cet « impensé d'une discipline qui ne cessait de l'utiliser comme un instrument taxinomique ", selon le mot de Michel de Certeau. Dans ses cheminements, Fr. Hartog a choisi d'accompagner quelques arpenteurs eux-mêmes spécialistes des traversées et des voyages imprévus. Au premier rang desquels, nous l'avons souligné, Ulysse et Chateaubriand. Les analyses savantes et subtiles dont ils sont l'objet sont sous-tendues par une ligne mélodique, une sorte de musique intérieure, qui est une interrogation sur l'histoire conçue comme déplacement incessant et traversée, comme voyage pour voir et savoir. Or cette conception désigne Hérodote comme le troisième héros de cette enquête sur le temps (p. 29), un Hérodote qui se présente ainsi, dès le début de sa propre Enquête (iotopín), sous les traits de Solon, dont la sagesse (бoధín) est associée à

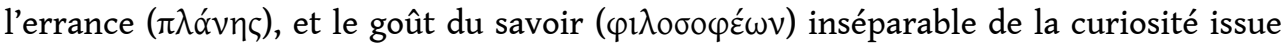
de la vue ( $\theta \varepsilon \omega p i ́ n \varsigma: ~ I, 30)$. Un Hérodote-Solon qui refuse de choisir définitivement entre les deux rives de l'Égée, entre Athènes et la Lydie des Barbares, parce qu'il sait que, de ce refus, dépendent sa véritable identité et le sens de ce régime de parole singulier, qui s'esquisse, qui n'a pas encore de nom, et que nous appelons désormais histoire. Et qui a pour mission d'ordonner et de penser, à partir du présent, les rapports qu'entretiennent les sociétés, d'ici et d'ailleurs, avec le passé et le futur.

\section{AUTEURS}

\section{PASCAL PAYEN}

Université de Toulouse-Le Mirail

payen@univ-tlse2.fr 